\title{
ON STABLE FULL-ORDER AND REDUCED-ORDER LQG CONTROLLERS
}

\author{
YORAM HALEVI \\ Faculty of Mechanical Engineering, Technion-Israel Institute of Technology, Haifa 3200, Israel \\ DENNIS S. BERNSTEIN \\ Harris Corporation, Government Aerospace Systems Division, Melbourne, FL 32902, U.S.A. \\ AND \\ WASSIM M. HADDAD \\ Department of Mechanical and Aerospace Engineering, Florida Institute of Technology, Melbourne, \\ FL 32902, U.S.A.
}

\begin{abstract}
SUMMARY
The problem of deriving a suboptimal LQG controller which is internally stable is considered. In the case where the controller is internally unstable a procedure is given for modifying the optimization parameters, i.e. the state weighting matrix and/or the process noise intensity. Sufficient conditions for controller stability of appropriately modified optimization problems are given for both the full-order and reduced-order cases. A final tuning procedure is then used to minimize the deviation from the original LQG problem.

KEY WORDS Optimal control LQG regulators Order reduction Stability
\end{abstract}

\section{INTRODUCTION}

One of the major approaches to feedback design is the linear quadratic Gaussian (LQG) optimization procedure. Except for the selection of weighting matrices for the mean square error criterion and stochastic models for the disturbances and measurement noise, the design of LQG controllers is automatic. These parameters together with the system parameters constitute the required data for well-known procedures of deriving the optimal output control law. In addition, the resulting closed loop is (under mild conditions) asymptotically stable. These two attractive properties are probably the main reasons for the popularity of LQG designs.

The LQG problem can be stated and solved either in the frequency domain or in the state space. In the frequency domain the optimal closed-loop transfer function is sought and the problem is formulated such that the Wiener-Hopf technique for optimal filtering may be employed. The optimal controller is then calculated from the optimal closed-loop transfer function. In the state space the solution is based on the separation principle, which states that the optimal controller is a combination of the optimal observer (the Kalman filter) and the optimal state feedback. The optimal controller does not appear explicitly in both solutions and

0143-2087/91/030163-10\$05.00

(c) 1991 by John Wiley \& Sons, Ltd.

Received 5 November 1990

Revised 22 August 1991 
no constraints are imposed on its structure and properties. Thus the optimal controller may be unstable even in cases where the plant is stable and minimum phase.

Theoretically the stability of the controller is irrelevant in the design of feedback systems since the closed-loop transfer function is the one that dictates the behaviour of the system. It is also well known that there are cases in which an unstable plant can be stabilized only by means of an unstable controller. ${ }^{1}$ However, if the plant is originally stable, then in practice a designer would be reluctant to use an unstable controller even if the latter is the outcome of an optimization and even if the stability margins are satisfactory. Among other reasons this is because the system then is always conditionally stable and becomes unstable when the gains of the plant or the sensor decrease substantially. One such case is simultaneous failure or saturation of the sensors in all loops. The system operates then in open loop and an unstable controller would lead to unbounded response.

In recent years necessary conditions for reduced-order estimators ${ }^{2}$ and compensators ${ }^{3}$ were given by means of the optimal projection equations. Those results were extended in several directions to include, for example, singular cases ${ }^{4-6}$ and $H_{\infty}$-bounds. ${ }^{7}$ In this method the order of the estimator or the controller is preconstrained and its parameters, namely the various matrices of its state space realization, are optimized with respect to the quadratic cost function. In the optimal compensation problem the solution consists of a set of four matrix equations, two modified Riccati equations and two modified Lyapunov equations, coupled by a projection matrix. As in the full-order case, the optimal closed loop is stable but internal stability of the controller is not guaranteed.

Despite its practical importance, the problem of stable LQG controllers has not received wide interest. A straightforward approach would be to solve a constrained parameter optimization problem where, in addition to closed-loop stability, stability of the controller is required. This problem was solved for a single-input/single-output system using the feasible direction approach. ${ }^{8}$ The order of the controller in this method is independent of that of the plant, so full-order, reduced-order and even extended-order optimal stable controllers can be found. As the order of the system and/or the number of inputs and outputs increase, this approach becomes numerically tedious. A more sophisticated approach, though eventually leading also to non-linear constrained parameter optimization, was given by Ganesh and Pearson. ${ }^{9}$ The order of the controller necessarily increases using that approach.

A different approach is to remain within the framework of the standard LQG problem and to achieve the internal stability of the controller by modifying the optimization parameters, i.e. the weighting matrices and the noise intensities. ${ }^{10}$ In the case where the required modification is relatively small the resulting controller is suboptimal with respect to the original problem. This approach is adopted in this paper. Sufficient conditions for stable, stabilizing LQG controllers for both the full-order and reduced-order cases are derived. After a stable LQG controller has been found, a final tuning procedure is used to find the minimal required change in that direction.

The paper is organized as follows. In Section 2 a stable suboptimal controller for the fullorder case is considered. Similar results for the reduced-order case are given in Section 3. Section 4 gives two illustrative examples. The results are discussed in Section 5.

\section{FULL-ORDER COMPENSATION}

Consider the $n$ th-order plant

$$
\begin{gathered}
\dot{\mathbf{x}}=\mathbf{A x}+\mathbf{B u}+\mathbf{w}_{1} \\
\mathbf{y}=\mathbf{C} \mathbf{x}+\mathbf{w}_{2}
\end{gathered}
$$


where $\mathbf{w}_{1}$ and $\mathbf{w}_{2}$ are mutually independent white noise processes having intensities $\mathbf{V}_{1} \geqslant 0$ and $\mathbf{V}_{2}>0$ respectively. The performance index is given by

$$
J=\lim _{t \rightarrow \infty} \mathrm{E}\left\{\mathbf{x}^{\mathrm{T}}(t) \mathbf{R}_{1} \mathbf{x}(t)+\mathbf{u}^{\mathrm{T}}(t) \mathbf{R}_{2} \mathbf{u}(t)\right\}, \quad \mathbf{R}_{1} \geqslant 0, \quad \mathbf{R}_{2}>0
$$

It is assumed that the pairs $(\mathbf{A}, \mathbf{B})$ and $\left(\mathbf{A}, \mathbf{V}_{1}^{1 / 2}\right)$ are stabilizable and the pairs $(\mathbf{A}, \mathbf{C})$ and $\left(\mathbf{A}, \mathbf{R}_{1}^{1 / 2}\right)$ are detectable. Since we focus mainly on stable plants, these requirements are satisfied automatically.

The optimal controller is given by ${ }^{11}$

$$
\begin{gathered}
\dot{\hat{\mathbf{x}}}=(\mathbf{A}-\mathbf{B F}-\mathbf{K C}) \hat{\mathbf{x}}+\mathbf{K} \mathbf{y} \\
\mathbf{u}=-\mathbf{F} \hat{\mathbf{x}}
\end{gathered}
$$

where

$$
\begin{aligned}
\mathbf{F} & =\mathbf{R}_{2}^{-1} \mathbf{B}^{\mathrm{T}} \mathbf{P} \\
\mathbf{K} & =\mathbf{Q C}^{\mathrm{T}} \mathbf{V}_{2}^{-1}
\end{aligned}
$$

and $\mathbf{P}$ and $\mathbf{Q}$ are the non-negative definite solutions of

$$
\begin{gathered}
\mathbf{P A}+\mathbf{A}^{\mathrm{T}} \mathbf{P}-\mathbf{P} \Sigma_{\mathrm{P}} \mathbf{P}+\mathbf{R}_{1}=0 \\
\mathbf{A} \mathbf{Q}+\mathbf{Q A}^{\mathrm{T}}-\mathbf{Q} \Sigma_{\mathrm{Q}} \mathbf{Q}+\mathbf{V}_{1}=0 \\
\Sigma_{\mathrm{P}} \triangleq \mathbf{B R}_{2}^{-1} \mathbf{B}^{\mathrm{T}}, \quad \Sigma_{\mathrm{Q}} \triangleq \mathbf{C}^{\mathrm{T}} \mathbf{V}_{2}^{-1} \mathbf{C}
\end{gathered}
$$

The following results are well known. ${ }^{11}$

(i) The eigenvalues of the closed-loop system are those of $\mathbf{A}-\mathbf{B F}$ and $\mathbf{A}-\mathbf{K C}$.

(ii) $\mathbf{A}-\mathbf{B F}$ and $\mathbf{A}-\mathbf{K C}$ are stability matrices, i.e. all of their eigenvalues lie in the open left half-plane (OLHP).

(iii) No stability properties of $\mathbf{A}-\mathbf{B F}-\mathbf{K C}$ are guaranteed.

In the case where the compensator is unstable the following sufficient condition may be used.

\section{Theorem 1}

Suppose $\mathbf{P}$ and $\mathbf{Q}$ are non-negative definite solutions of

$$
\begin{gathered}
\mathbf{A} \mathbf{Q}+\mathbf{Q} \mathbf{A}^{\mathrm{T}}-\mathbf{Q} \Sigma_{\mathbf{Q}} \mathbf{Q}+\mathbf{V}_{1}=0 \\
\mathbf{P A}+\mathbf{A}^{\mathrm{T}} \mathbf{P}-\mathbf{P} \Sigma_{\mathbf{P}} \mathbf{P}+\mathbf{R}_{1}+\left(\rho \mathbf{P}-\rho^{-1} \Sigma_{\mathbf{Q}} \mathbf{Q}^{1-\alpha}\right) \mathbf{Q}^{\alpha}\left(\rho \mathbf{P}-\rho^{-1} \Sigma_{\mathbf{Q}} \mathbf{Q}^{1-\alpha}\right)^{\mathrm{T}}=0
\end{gathered}
$$

for some scalars $\rho$ and $\alpha$. Then $\mathbf{A}-\mathbf{B F}-\mathbf{K C}$, whereas $\mathbf{F}$ and $\mathbf{K}$ are defined in (6) and (7), is a stability matrix and the controller (4), (5) stabilizes the plant (1), (2).

Proof. Equation (11) may be rearranged, using $\mathbf{Q} \Sigma_{\mathbf{Q}}=\mathbf{K C}$, as

$$
\mathbf{P}(\mathbf{A}-\mathbf{K C})+(\mathbf{A}-\mathbf{K C})^{\mathrm{T}} \mathbf{P}-\mathbf{P} \Sigma_{\mathbf{P}} \mathbf{P}+\mathbf{R}_{1}+\rho^{2} \mathbf{P} \mathbf{Q}^{\alpha} \mathbf{P}+\rho^{-2} \Sigma_{\mathrm{Q}} \mathbf{Q}^{2-\alpha} \Sigma_{\mathbf{Q}}=0
$$

This is a Riccati equation for the system $(\mathbf{A}-\mathbf{K C}, \mathbf{B})$ with a state weighting matrix $\tilde{\mathbf{R}} \triangleq \mathbf{R}_{1}+\rho^{2} \mathbf{P} \mathbf{Q}^{\alpha} \mathbf{P}+\rho^{-2} \Sigma_{Q} \mathbf{Q}^{2-\alpha} \Sigma_{Q} \geqslant 0$. Since $\mathbf{A}-\mathbf{K C}$ is asymptotically stable, all stabilizability and detectability conditions are met. Using (ii), $\mathbf{A}-\mathbf{K C}-\mathbf{B F}$ is asymptotically stable. The resulting controller is stabilizing since it is optimal with respect to the LQG problem (3) with $\mathbf{R}_{1}$ modified by a non-negative definite matrix (the last term of (11)) and the same $\mathbf{R}_{2}$. 


\section{Remark 1}

A dual result can be easily obtained by fixing the state weighting matrix $\mathbf{R}_{1}$, which is the only parameter that was changed, and modifying $\mathbf{V}_{1}$ in a similar fashion.

For its numerical solution it is convenient to have (11) in the form of (12) with the quadratic terms in $\mathbf{P}$ lumped together to give an indefinite term. $-\mathbf{P}\left(\Sigma_{\mathbf{P}}-\rho^{2} \mathbf{Q}^{\alpha}\right) \mathbf{P}$. This type of Riccati equation is encountered in $H_{\infty}$-theory. It is solved via the eigenvector matrix of the Hamiltonian matrix ${ }^{11}$

$$
\mathbf{H}=\left[\begin{array}{cc}
\tilde{\mathbf{A}} & \tilde{\Sigma} \\
-\tilde{\mathbf{Q}} & -\tilde{\mathbf{A}}^{\mathrm{T}}
\end{array}\right]
$$

where $\tilde{\mathbf{A}}=\mathbf{A}-\mathbf{K C}, \tilde{\boldsymbol{\Sigma}}=-\left(\boldsymbol{\Sigma}_{\mathrm{P}}-\rho^{2} \mathbf{Q}^{\alpha}\right)$ and $\tilde{\mathbf{Q}}=\mathbf{R}_{1}+\rho^{-2} \Sigma_{\mathrm{Q}} \mathbf{Q}^{2-\alpha} \boldsymbol{\Sigma}_{\mathrm{Q}}$. Since $\tilde{\boldsymbol{\Sigma}}$ is indefinite, existence of a non-negative definite solution $\mathbf{P}$ is not guaranteed and it depends on the value of $\rho$. When $\rho \rightarrow 0, \tilde{\Sigma} \rightarrow-\Sigma_{\mathrm{P}}$ and then a solution exists. However, in that case $\tilde{\mathbf{Q}}$ becomes very large. We therefore want to use the maximum possible $\rho$ and search for it by increasing $\rho$ until a pair of eigenvalues of $\mathbf{H}$ reach the imaginary axis.

Theorem 1 is a sufficient condition for the stability of the controller and as such does not give the minimal modification required. Several final tuning algorithms may be used to minimize the change in $\mathbf{R}_{1}$. One way is to solve (8) with $\mathbf{R}_{1}$ replaced by

$$
\overline{\mathbf{R}}(\lambda)=\lambda \tilde{\mathbf{R}}+(1-\lambda) \mathbf{R}_{1}=\mathbf{R}_{1}+\lambda\left(\rho \overline{\mathbf{P}}-\rho^{-1} \Sigma_{\mathbf{Q}} \mathbf{Q}^{1-\alpha}\right) \mathbf{Q}^{\alpha}\left(\rho \overline{\mathbf{P}}-\rho^{-1} \Sigma_{\mathbf{Q}} \mathbf{Q}^{1-\alpha}\right)^{\mathrm{T}}
$$

where $\overline{\mathbf{P}}$ is the solution of (11). The controller poles are continuous with respect to $\mathbf{P}(\lambda)$, which is continuous itself, hence they are continuous with respect to $\lambda$. For $\lambda=0$, where the controller is unstable, at least one of these poles is in the right half-plane (RHP), and for $\lambda=1$, where it is strictly stable, all of them are in the OLHP. From continuity considerations there exists $\lambda^{*}<1$ such that the resulting controller is stable for all $1>\lambda>\lambda^{*}$. (In general there might be several crossings of the imaginary axis and $\lambda^{*}$ corresponds to the last one. Therefore it is sometimes possible to find and use $\lambda<\lambda^{*}$, which leads to a stable controller.) A slightly different procedures is to replace (11) by

$$
\mathbf{P A}+\mathbf{A}^{\mathrm{T}} \mathbf{P}-\mathbf{P} \Sigma_{\mathbf{P}} \mathbf{P}+\mathbf{R}_{1}+\lambda\left(\rho \mathbf{P}-\rho^{-1} \Sigma_{\mathbf{Q}} \mathbf{Q}^{1-\alpha}\right) \mathbf{Q}^{\alpha}\left(\rho \mathbf{P}-\rho^{-1} \Sigma_{\mathrm{Q}} \mathbf{Q}^{1-\alpha}\right)^{\mathrm{T}}=0
$$

In this formulation we have an equation similar to (14) which is solved using the same method. Again $\lambda$ is increased until a stable controller is reached. Note that the two procedures are not identical and coincide only for $\lambda=0$ and $\lambda=1$.

In general the existence of $\rho$ and $\alpha$ that lead to $\mathbf{P} \geqslant 0$ is not guaranteed. For $\rho \rightarrow 0$ or $\alpha \rightarrow-\infty$ the additional quadratic terms in $\mathbf{P}$ seems negligible; however, this makes the last term of (12) infinite and consequently the quadratic terms in $\mathbf{P}$ in that equation dominate the solution. Allowing another degree of freedom, the following lemma guarantees the existence of a stable LQG compensator.

\section{Lemma 2}

Assume that $\mathbf{A}$ is asymptotically stable and that $\left(\mathbf{A}, \mathbf{R}_{1}^{1 / 2}\right)$ is observable. Then there exist $\varepsilon>0, \alpha$ and $\rho$ such that the equations

$$
\begin{gathered}
\mathbf{A} \mathbf{Q}+\mathbf{Q} \mathbf{A}^{\mathrm{T}}-\mathbf{Q} \Sigma_{\mathrm{Q}} \mathbf{Q}+\varepsilon \mathbf{V}_{1}=0 \\
\mathbf{P} \mathbf{A}+\mathbf{A}^{\mathrm{T}} \mathbf{P}-\mathbf{P} \Sigma_{\mathbf{P}} \mathbf{P}+\mathbf{R}_{1}+\left(\rho \mathbf{P}-\rho^{-1} \Sigma_{\mathbf{Q}} \mathbf{Q}^{1-\alpha}\right) \mathbf{Q}^{\alpha}\left(\rho \mathbf{P}-\rho^{-1} \Sigma_{Q} \mathbf{Q}^{1-\alpha}\right)^{\mathrm{T}}=0
\end{gathered}
$$

have non-negative definite solutions $\mathbf{Q}$ and $\mathbf{P}$. 
Proof. Let $\mathbf{Q}_{1}$ be the solution of the Lyapunov equation

$$
\mathbf{A} \mathbf{Q}_{1}+\mathbf{Q}_{1} \mathbf{A}^{\mathrm{T}}+\mathbf{V}_{1}=0
$$

Since $\mathbf{A}$ is asymptotically stable, $\mathbf{Q}_{1} \geqslant 0$. Then as $\varepsilon$ approaches zero, $\mathbf{Q}=\varepsilon \mathbf{Q}_{1}+o(\varepsilon)$. Now with $\alpha=0$ and $\rho=\varepsilon^{1 / 2}$ equation (15) becomes

$$
\mathbf{P A}+\mathbf{A}^{\mathrm{T}} \mathbf{P}-\mathbf{P} \Sigma_{\mathrm{P}} \mathbf{P}+\mathbf{R}_{1}+\varepsilon^{1 / 2}\left(\mathbf{P}-\Sigma_{\mathrm{Q}} \mathbf{Q}_{1}\right)\left(\mathbf{P}-\mathbf{Q}_{1} \Sigma_{\mathbf{Q}}\right)+o\left(\varepsilon^{1 / 2}\right)=0
$$

Observability of $\left(\mathbf{A}, \mathbf{R}_{1}^{1 / 2}\right)$ implies that for $\varepsilon=0$, i.e. the original Riccati equation, $\mathbf{P}$ is positive definite. From continuity the solution of (16) with sufficiently small $\varepsilon$ is also positive definite.

\section{REDUCED-ORDER COMPENSATION}

The reduced-order optimal control problem is stated as follows.

For the plant (1), (2) find an $n_{\mathrm{c}}$ th-order compensator

$$
\begin{gathered}
\dot{\mathbf{x}}_{\mathrm{c}}=\mathbf{A}_{\mathrm{c}} \mathbf{x}_{\mathrm{c}}+\mathbf{B}_{\mathrm{c}} \mathbf{y} \\
\mathbf{u}=\mathbf{C}_{\mathrm{c}} \mathbf{x}_{\mathrm{c}}
\end{gathered}
$$

which minimizes the cost (3).

The solution of this problem, actually necessary conditions characterizing all optimal solutions, is summarized in the following theorem.

\section{Theorem $2^{3}$}

Suppose $\left(\mathbf{A}_{c}, \mathbf{B}_{\mathrm{c}}, \mathbf{C}_{\mathrm{c}}\right)$ solves the reduced-order optimal control problem. Then there exist $n \times n$ non-negative definite matrices $\mathbf{Q}, \mathbf{P}, \hat{\mathbf{Q}}, \hat{\mathbf{P}}$ such that $\mathbf{A}_{\mathrm{c}}, \mathbf{B}_{\mathrm{c}}$ and $\mathbf{C}_{\mathrm{c}}$ are given by

$$
\begin{gathered}
\mathbf{A}_{\mathrm{c}}=\Gamma\left(\mathbf{A}-\mathbf{Q} \Sigma_{\mathrm{Q}}-\Sigma_{\mathrm{P}} \mathbf{P}\right) \mathbf{G}^{\mathrm{T}} \\
\mathbf{B}_{\mathrm{c}}=\Gamma \mathbf{Q} \mathbf{C}^{\mathrm{T}} \mathbf{V}_{2}^{-1} \\
\mathbf{C}_{\mathrm{c}}=-\mathbf{R}_{2}^{-1} \mathbf{B}^{\mathrm{T}} \mathbf{P} \mathbf{G}^{\mathrm{T}}
\end{gathered}
$$

where $\mathbf{Q}, \mathbf{P}, \hat{\mathbf{Q}}, \hat{\mathbf{P}}, \Gamma$ and $\mathbf{G}^{\mathrm{T}}$ satisfy

$$
\begin{gathered}
\mathbf{A} \mathbf{Q}+\mathbf{Q} \mathbf{A}^{\mathrm{T}}+\mathbf{V}_{1}-\mathbf{Q} \Sigma_{\mathrm{Q}} \mathbf{Q}+\tau_{\perp} \mathbf{Q} \Sigma_{\mathrm{Q}} \mathbf{Q} \tau_{\perp}^{\mathrm{T}}=0 \\
\left(\mathbf{A}-\Sigma_{\mathrm{P}} \mathbf{P}\right) \hat{\mathbf{Q}}+\hat{\mathbf{Q}}\left(\mathbf{A}-\Sigma_{\mathrm{P}} \mathbf{P}\right)^{\mathrm{T}}+\mathbf{Q} \Sigma_{\mathrm{Q}} \mathbf{Q}-\tau_{\perp} \mathbf{Q} \Sigma_{\mathrm{Q}} \mathbf{Q} \tau_{\perp}^{\mathrm{T}}=0 \\
\mathbf{A}^{\mathrm{T}} \mathbf{P}+\mathbf{P} \mathbf{A}+\mathbf{R}_{1}-\mathbf{P} \Sigma_{\mathrm{P}} \mathbf{P}+\boldsymbol{\tau}_{\perp}^{\mathrm{T}} \mathbf{P} \Sigma_{\mathrm{P}} \mathbf{P} \tau_{\perp}=0 \\
\hat{\mathbf{P}}\left(\mathbf{A}-\mathbf{Q} \Sigma_{\mathrm{Q}}\right)+\left(\mathbf{A}-\mathbf{Q} \Sigma_{\mathrm{Q}}\right)^{\mathrm{T}} \hat{\mathbf{P}}+\mathbf{P} \Sigma_{\mathrm{P}} \mathbf{P}-\tau_{\perp}^{\mathrm{T}} \mathbf{P} \Sigma_{\mathrm{P}} \mathbf{P} \tau_{\perp}=0 \\
\operatorname{rank} \hat{\mathbf{Q}}=\operatorname{rank} \hat{\mathbf{P}}=\operatorname{rank} \hat{\mathbf{Q}} \hat{\mathbf{P}}=n_{\mathrm{c}} \\
\hat{\mathbf{Q}} \hat{\mathbf{P}}=\mathbf{G}^{\mathrm{T}} \mathbf{M} \Gamma, \quad \quad \mathbf{\Gamma G}^{\mathrm{T}}=\mathbf{I}_{n_{\mathrm{c}}}, \quad \mathbf{M}\left(n_{\mathrm{c}} \times n_{\mathrm{c}}\right) \text { non-singular } \\
\tau=\mathbf{G}^{\mathrm{T}} \Gamma, \quad \tau_{\perp}=\mathbf{I}_{n}-\tau
\end{gathered}
$$

As in the full-order case, the optimal reduced-order controller (if exists) stabilizes the system but may be unstable itself. To derive a stable controller, we use an approach similar to the one used in the full-order case. The optimization parameters, or equivalently equations (22)-(25), are modified and known stability results of a partial problem, LQ or optimal estimation, are employed. We therefore start by stating the reduced-order optimal estimation problem. 
Given the system (1), (2), which is assumed now to be asymptotically stable, and the criterion

$$
J_{1}=\lim _{t \rightarrow \infty} \mathrm{E}\left\{\left(\mathbf{L} \mathbf{x}-\mathbf{y}_{\mathrm{e}}\right)^{\mathrm{T}} \mathbf{R}\left(\mathbf{L} \mathbf{x}-\mathbf{y}^{\mathrm{e}}\right)\right\}
$$

where $\mathbf{L}$ is a constant matrix representing linear combinations of the states that are to be estimated and $\mathbf{R}>0$ is a weighting matrix, find an $n_{\mathrm{e}}$ th-order estimator

$$
\begin{gathered}
\dot{\mathbf{x}}_{\mathrm{e}}=\mathbf{A}_{\mathrm{e}} \mathbf{X}_{\mathrm{e}}+\mathbf{B}_{\mathrm{e}} \mathbf{y} \\
\mathbf{y}_{\mathrm{e}}=\mathbf{C}_{\mathrm{e}} \mathbf{x}_{\mathrm{e}}
\end{gathered}
$$

which minimizes (29).

The following theorem gives necessary conditions for the reduced-order optimal estimator.

\section{Theorem $3^{2}$}

Suppose ( $\mathbf{A}_{\mathrm{c}}, \mathbf{B}_{\mathrm{c}}, \mathbf{C}_{\mathrm{e}}$ ) solves the reduced-order optimal estimation problem. Then $\mathbf{A}_{\mathrm{e}}, \mathbf{B}_{\mathrm{e}}$ and $\mathbf{C}_{\mathrm{e}}$ are given by

$$
\begin{gathered}
\mathbf{A}_{\mathrm{e}}=\Gamma\left(\mathbf{A}-\mathbf{Q} \Sigma_{\mathrm{Q}}\right) \mathbf{G}^{\mathrm{T}} \\
\mathbf{B}_{\mathrm{e}}=\Gamma \mathbf{Q} \mathbf{C}^{\mathrm{T}} \mathbf{V}_{2}^{-1} \\
\mathbf{C}_{\mathrm{e}}=\mathbf{L} \mathbf{G}^{\mathrm{T}}
\end{gathered}
$$

where $\mathbf{Q}, \hat{\mathbf{Q}}$ and $\hat{\mathbf{P}}$ are non-negative definite matrices satisfying

$$
\begin{gathered}
\mathbf{A} \mathbf{Q}+\mathbf{Q} \mathbf{A}^{\mathrm{T}}+\mathbf{V}_{1}-\mathbf{Q} \Sigma_{\mathrm{Q}} \mathbf{Q}+\tau_{\perp} \mathbf{Q} \Sigma_{\mathrm{Q}} \mathbf{Q} \tau_{\perp}^{\mathrm{T}}=0 \\
\mathbf{A} \hat{\mathbf{Q}}+\hat{\mathbf{Q}} \mathbf{A}^{\mathrm{T}}+\mathbf{Q} \Sigma_{\mathrm{Q}} \mathbf{Q}-\tau_{\perp} \mathbf{Q} \Sigma_{\mathrm{Q}} \mathbf{Q} \tau_{\perp}^{\mathrm{T}}=0 \\
\hat{\mathbf{P}}\left(\mathbf{A}-\mathbf{Q} \Sigma_{\mathbf{Q}}\right)+\left(\mathbf{A}-\mathbf{Q} \Sigma_{\mathrm{Q}}\right)^{\mathrm{T}} \hat{\mathbf{P}}+\mathbf{L}^{\mathrm{T}} \mathbf{R} \mathbf{L}-\tau_{\perp}^{\mathrm{T}} \mathbf{L}^{\mathrm{T}} \mathbf{R} \mathbf{L} \tau_{\perp}=0
\end{gathered}
$$

and $\mathbf{G}^{\mathrm{T}}, \Gamma, \tau$ and $\tau_{\perp}$ are as in (26)-(28) with $n_{\mathrm{e}}$ replacing $n_{\mathrm{c}}$.

Certainly if an optimal estimator exists, it is asymptotically stable. We are now in a position to state the main result of this section, which gives a sufficient condition for the existence of a stable, stabilizing suboptimal reduced-order controller.

\section{Theorem 4}

Suppose $\mathbf{Q}, \mathbf{P}, \hat{\mathbf{Q}}$ and $\hat{\mathbf{P}}$ are non-negative definite matrices satisfying

$$
\begin{aligned}
& \mathbf{A} \mathbf{Q}+\mathbf{Q} \mathbf{A}^{\mathrm{T}}-\mathbf{Q} \Sigma_{\mathrm{Q}} \mathbf{Q}+\tau_{\perp} \mathbf{Q} \Sigma_{\mathrm{Q}} \mathbf{Q} \tau_{\perp}^{\mathrm{T}}+\mathbf{V}_{\perp} \\
& +\left(\rho_{1} \mathbf{Q}-\rho_{1}^{-1} \Sigma_{\mathbf{P}} \mathbf{P}^{1-\alpha}\right) \mathbf{P}^{\alpha}\left(\rho_{1} \mathbf{Q}-\rho_{1}^{-1} \Sigma_{\mathbf{P}} \mathbf{P}^{1-\alpha}\right)^{\mathrm{T}}=0 \\
& \left(\mathbf{A}-\Sigma_{\mathbf{P}} \mathbf{P}\right) \hat{\mathbf{Q}}+\hat{\mathbf{Q}}\left(\mathbf{A}-\Sigma_{\mathrm{P}} \mathbf{P}\right)^{\mathrm{T}}+\mathbf{Q} \Sigma_{\mathrm{Q}} \mathbf{Q}-\tau_{\perp} \mathbf{Q} \Sigma_{\mathrm{Q}} \mathbf{Q} \tau_{\perp}^{\mathrm{T}}=0 \\
& \mathbf{P A}+\mathbf{A}^{\mathrm{T}} \mathbf{P}-\mathbf{P} \Sigma_{\mathbf{P}} \mathbf{P}+\tau_{\perp}^{\mathrm{T}} \mathbf{P} \Sigma_{\mathrm{P}} \mathbf{P} \tau_{\perp}+\mathbf{R}_{1}+\left(\rho_{2} \mathbf{P}-\rho_{2}^{-1} \hat{\mathbf{P}}\right) \Sigma_{\mathrm{P}}\left(\rho_{2} \mathbf{P}-\rho_{2}^{-1} \hat{\mathbf{P}}\right) \\
& +\rho_{3}^{-2} \mathbf{A}^{\mathrm{T}} \mathbf{A}+\rho_{3}^{2} \mathbf{P}_{2}=0 \\
& \hat{\mathbf{P}}\left(\mathbf{A}-\mathbf{Q} \Sigma_{\mathrm{Q}}\right)+\left(\mathbf{A}-\mathrm{Q} \Sigma_{\mathrm{Q}}\right)^{\mathrm{T}} \hat{\mathbf{P}}+\mathbf{P} \Sigma_{\mathrm{P}} \mathbf{P}-\tau_{\perp}^{\mathrm{T}} \mathbf{P} \Sigma_{\mathrm{P}} \mathbf{P} \tau_{\perp}=0 \\
& \operatorname{rank} \hat{\mathbf{Q}}=\operatorname{rank} \hat{\mathbf{P}}=\operatorname{rank} \hat{\mathbf{Q}} \hat{\mathbf{P}}=n_{\mathrm{C}} \\
& \hat{\mathbf{Q}} \hat{\mathbf{P}}=\mathbf{G}^{\mathrm{T}} \mathbf{M} \Gamma, \quad \boldsymbol{\Gamma G}^{\mathrm{T}}=\mathbf{I}_{n_{\mathrm{c}}}, \quad \mathbf{M}\left(n_{\mathrm{c}} \times n_{\mathrm{c}}\right) \text { non-singular } \\
& \tau=\mathbf{G}^{\mathrm{T}} \Gamma, \quad \tau_{\perp}=\mathbf{I}_{n}-\tau
\end{aligned}
$$


for some $\rho_{1}, \rho_{2}, \rho_{3}$ and $\alpha$. Then the controller (17), (18) with

$$
\begin{aligned}
\mathbf{A}_{\mathrm{c}}=\Gamma\left(\mathbf{A}-\mathbf{Q} \Gamma_{\mathrm{Q}}-\Gamma_{\mathrm{P}}\right) \mathbf{G}^{\mathrm{T}} \\
\mathbf{B}_{\mathrm{c}}=\Gamma \mathbf{Q} \mathbf{C}^{\mathrm{T}} \mathbf{V}_{2}^{-1} \\
\mathbf{C}_{\mathrm{c}}=\mathbf{R}_{2}^{-1} \mathbf{B}^{\mathrm{T}} \mathbf{P} \mathbf{G}^{\mathrm{T}}
\end{aligned}
$$

is asymptotically stable and stabilizes the system (1), (2).

Proof. To show that the compensator is stabilizing, note that the equations have the form of (22)-(25), the necessary conditions for the optimal reduced-order compensator for the original system, with

$$
\begin{gathered}
\overline{\mathbf{R}}_{1}=\mathbf{R}_{1}+\left(\rho_{2} \mathbf{P}-\rho_{2}^{-1} \hat{\mathbf{P}}\right) \Sigma_{\mathrm{P}}\left(\rho_{2} \mathbf{P}-\rho_{2}^{-1} \hat{\mathbf{P}}\right)+\rho_{3}^{-2} \mathbf{A}^{\mathrm{T}} \mathbf{A}+\rho_{3}^{2} \mathbf{P}^{2} \geqslant 0 \\
\overline{\mathbf{V}}_{1}=\mathbf{V}_{1}+\left(\rho_{1} \mathbf{Q}-\rho_{1}^{-1} \Sigma_{\mathbf{P}} \mathbf{P}^{1-\alpha}\right) \mathbf{P}^{\alpha}\left(\rho_{1} \mathbf{Q}-\rho_{1}^{-1} \Sigma_{\mathbf{P}} \mathbf{P}^{1-\alpha}\right)^{\mathrm{T}} \geqslant 0
\end{gathered}
$$

replacing $\mathbf{R}_{1}$ and $\mathbf{V}_{1}$ respectively.

Consider now equation (40), which can be written as

$$
\begin{aligned}
\mathbf{P} \Sigma_{\mathrm{P}} \mathbf{P}+\mathbf{P} \Sigma_{\mathrm{P}} \hat{\mathbf{P}}+\hat{\mathbf{P}} \Sigma_{\mathrm{P}} \mathbf{P}=\left(\rho_{3}^{-1} \mathbf{A}+\rho_{3} \mathbf{P}\right)^{\mathrm{T}}\left(\rho_{3}^{-1} \mathbf{A}+\rho_{3} \mathbf{P}\right)+\tau_{\perp}^{\mathrm{T}} \mathbf{P} \Sigma_{\mathrm{P}} \mathbf{P} \tau_{\perp}+\mathbf{R}_{1} \\
+\rho_{2}^{2} \mathbf{P} \Sigma_{\mathbf{P}} \mathbf{P}+\rho_{2}^{-2} \hat{\mathbf{P}} \Sigma_{\mathrm{P}} \hat{\mathbf{P}}
\end{aligned}
$$

Since the right-hand side of (50) is non-negative definite, there exist $\mathbf{R}>0$ and $\mathbf{L}$ such that

$$
\mathbf{P} \Sigma_{\mathrm{P}} \mathbf{P}+\mathbf{P} \Sigma_{\mathrm{P}} \hat{\mathbf{P}}+\hat{\mathbf{P}} \Sigma_{\mathrm{P}} \mathbf{P}=\mathbf{L}^{\mathrm{T}} \mathbf{R} \mathbf{L}
$$

An important relation in the development of the optimal projection equations ${ }^{2-7}$ is $\hat{\mathbf{P}} \tau=\hat{\mathbf{P}}$ which implies $\hat{\mathbf{P}} \tau_{\perp}=0$. Subtracting the identically zero term $\tau_{\perp}^{\mathrm{T}}\left(\hat{\mathbf{P}} \Sigma_{\mathrm{P}} \mathbf{P}+\mathbf{P} \Sigma_{\mathrm{P}} \hat{\mathbf{P}}\right) \tau_{\perp}$ from (41) using (51) yields

$$
\hat{\mathbf{P}}\left(\mathbf{A}-\Sigma_{\mathrm{P}} \mathbf{P}-\mathbf{Q} \Sigma_{\mathrm{Q}}\right)+\left(\mathbf{A}-\Sigma_{\mathrm{P}} \mathbf{P}-\mathbf{Q} \Sigma_{\mathrm{Q}}\right)^{\mathrm{T}} \hat{\mathbf{P}}+\mathbf{L}^{\mathrm{T}} \mathbf{R} \mathbf{L}-\tau_{\perp}^{\mathrm{T}} \mathbf{L}^{\mathrm{T}} \mathbf{R} \mathbf{L} \tau_{\perp}=0
$$

Rearranging (38), we get

$$
\left(\mathbf{A}-\Sigma_{\mathrm{P}} \mathbf{P}\right) \mathbf{Q}+\mathbf{Q}\left(\mathbf{A}-\Sigma_{\mathrm{P}} \mathbf{P}\right)^{\mathrm{T}}-\mathbf{Q} \Sigma_{\mathrm{Q}} \mathbf{Q}+\tau_{\perp} \mathbf{Q} \Sigma_{\mathrm{Q}} \mathbf{Q} \tau_{\perp}^{\mathrm{T}}+\tilde{\mathbf{V}}_{1}=0
$$

where

$$
\tilde{\mathbf{V}}_{1} \triangleq \mathbf{V}_{1}+\rho_{1}^{2} \mathbf{Q} \mathbf{P}^{\alpha} \mathbf{Q}+\rho_{1}^{-2} \Sigma_{\mathrm{P}} \mathbf{P}^{2-\alpha} \Sigma_{\mathrm{P}}
$$

Equation (53), (39) and (52) are analogous to (35)-(37) and constitute the optimal projection equations for a system with system matrix $\tilde{\mathbf{A}}=\mathbf{A}-\Sigma_{\mathrm{P}} \mathbf{P}$, process noise intensity $\tilde{\mathbf{V}}_{1}$, and $\mathbf{L}$ and $\mathbf{R}$ as defined in (51). To use the stability results of the reduced-order estimator, we still have to show first that $\tilde{\mathbf{A}}$ is stable. Defining

$$
\tilde{\mathbf{R}}_{1} \triangleq \mathbf{R}_{1}+\tau_{\perp}^{\mathrm{T}} \mathbf{P} \Sigma_{\mathrm{P}} \mathbf{P} \tau_{\perp}+\left(\rho_{2} \mathbf{P}-\rho_{2}^{-1} \hat{\mathbf{P}}\right) \Sigma_{\mathbf{P}}\left(\rho_{2} \mathbf{P}-\rho_{2}^{-1} \hat{\mathbf{P}}\right)+\rho_{3}^{-2} \mathbf{A}^{\mathrm{T}} \mathbf{A}+\rho_{3}^{2} \mathbf{P}^{2}
$$

equation (40) becomes

$$
\mathbf{P A}+\mathbf{A}^{\mathrm{T}} \mathbf{P}-\mathbf{P} \Sigma_{\mathbf{P}} \mathbf{P}+\tilde{\mathbf{R}}_{1}=0
$$

Since $\tilde{\mathbf{R}}_{1} \geqslant \mathbf{R}_{1},\left(\mathbf{A}, \mathbf{R}_{1}^{1 / 2}\right)$ detectable implies $\left(\mathbf{A}, \tilde{\mathbf{R}}_{1}^{1 / 2}\right)$ detectable, which from LQG theory ${ }^{11}$ implies $\mathbf{A}-\Sigma_{\mathrm{P}} \mathbf{P}$ asymptotically stable. Now the dynamics of the reduced-order estimator for the problem defined by (53), (39) and (52) is

$$
\tilde{\mathbf{A}}_{\mathrm{e}}=\Gamma\left(\tilde{\mathbf{A}}-\mathbf{Q} \Sigma_{\mathrm{Q}}\right) \mathbf{G}^{\mathrm{T}}=\Gamma\left(\mathbf{A}-\Sigma_{\mathrm{P}} \mathbf{P}-\mathbf{Q} \Sigma_{\mathrm{Q}}\right) \mathbf{G}^{\mathrm{T}}
$$

which is asymptotically stable. This completes the proof. 
The two final tuning procedures outlined in Section 2 are applicable here as well, with the distinction that in Theorem 4 both the process noise intensity and the state weighting matrix were modified. The final tuning may be applied using either a single parameter $\lambda$ for both modifications or two parameters $\lambda_{1}$ and $\lambda_{2}$ to allow uneven deviations from the original problem. The solution of (38)-(41) is not an easy task. The equations are coupled by the projection and their quadratic terms have indefinite coefficient matrices. The method which we used to solve these equations was repeated substitutions. Equations (38) and (40) were treated as standard Riccati equations where all the additional terms were evaluated from the previous iteration and added to the constant term. A final tuning scheme similar to (14) can be used as a simple ad hoc homotopy algorithm, which gives a good initial guess for the solution. Equation (38) and (40) are replaced by

$$
\begin{aligned}
\mathbf{A} \mathbf{Q}+\mathbf{Q} \mathbf{A}^{\mathrm{T}}-\mathbf{Q} \Sigma_{\mathrm{Q}} \mathbf{Q}+\tau_{\perp} \mathbf{Q} \Sigma_{\mathrm{Q}} \mathbf{Q} \tau_{\perp}^{\mathrm{T}}+\mathbf{V}_{1} \\
+\lambda_{1}\left(\rho_{1} \mathbf{Q}-\rho_{1}^{-1} \Sigma_{\mathrm{P}} \mathbf{P}^{1-\alpha}\right) \mathbf{P}^{\alpha}\left(\rho_{1} \mathbf{Q}-\rho_{1}^{-1} \Sigma_{\mathrm{P}} \mathbf{P}^{1-\alpha}\right)^{\mathrm{T}}=0 \\
\mathbf{P A}+\mathbf{A}^{\mathrm{T}} \mathbf{P}-\mathbf{P} \Sigma_{\mathrm{P}} \mathbf{P}+\tau_{\perp}^{\mathrm{T}} \mathbf{P} \Sigma_{\mathrm{P}} \mathbf{P} \tau_{\perp}+\mathbf{R}_{1} \\
+\lambda_{2}\left[\left(\rho_{2} \mathbf{P}-\rho_{2}^{-1} \hat{\mathbf{P}}\right) \Sigma_{\mathrm{P}}\left(\rho_{2} \mathbf{P}-\rho_{2}^{-1} \hat{\mathbf{P}}\right)+\rho_{3}^{-2} \mathbf{A}^{\mathrm{T}} \mathbf{A}+\rho_{3}^{2} \mathbf{P}^{2}\right]=0
\end{aligned}
$$

respectively. Starting with $\lambda_{1}=\lambda_{2}=0$, which is the optimal unstable case, and increasing $\lambda_{1}$ and $\lambda_{2}$ gradually while checking the stability of $\mathbf{A}_{\mathrm{c}}$ is a convenient way to solve these equations and to have final tuning at the same time.

\section{Example 1}

\section{EXAMPLES}

Consider the system

$$
\begin{aligned}
& \dot{\mathbf{x}}=\left[\begin{array}{cc}
0 & 1 \\
-3 & -4
\end{array}\right] \mathbf{x}+\left[\begin{array}{l}
0 \\
1
\end{array}\right] \mathbf{u}+\left[\begin{array}{r}
35 \\
-61
\end{array}\right] \mathbf{w} \\
& \mathbf{y}=\left[\begin{array}{ll}
2 & 1
\end{array}\right] \mathbf{x}+\mathbf{v}
\end{aligned}
$$

where $\mathbf{w}$ and $\mathbf{v}$ are unit-intensity white noise processes. The state and input weighting matrices are

$$
\mathbf{R}_{1}=\left[\begin{array}{rr}
2800 & 473 \\
473 & 80
\end{array}\right], \quad \mathbf{R}_{2}=1
$$

This example was first introduced by Doyle and Stein ${ }^{12}$ and was also considered in the context of stable compensators. ${ }^{8,9}$ The optimal compensator has an observer gain $\mathbf{K}=\left[\begin{array}{ll}30-50\end{array}\right]^{\mathrm{T}}$ and a state feedback $\mathbf{F}=\left[\begin{array}{ll}50 & 10\end{array}\right]$. Its transfer function is

$$
C(s)=\frac{1000(s+2 \cdot 6)}{s^{2}+24 s-797}
$$

which is unstable. The optimal cost is $2 \cdot 44 \times 10^{5}$. Using the method of Theorem 1 with $\alpha=0$ and $\rho=0.064$, we get the stable compensator

$$
C_{1}(s)=\frac{8 \cdot 76 \times 10^{3}(s+5 \cdot 1)}{s^{2}+922 s+2215}
$$

which yields the cost $4 \cdot 61 \times 10^{5}$. The final tuning described in (13) gives, with $\lambda=0 \cdot 013$,

$$
C_{2}(s)=\frac{1 \cdot 32 \times 10^{3}(s+4 \cdot 44)}{s(s+118 \cdot 2)}
$$


and the cost $4.06 \times 10^{5}$. This value is close to the minimum cost possible by a second-order stable compensator, which was found ${ }^{8}$ to be $3.95 \times 10^{5}$. Similar results were obtained by using the final tuning method of (14). It is worth noting that better performance can be achieved by increasing the order of the compensator. ${ }^{9}$

\section{Example 2}

Consider the system

$$
\begin{gathered}
\dot{\mathbf{x}}=\left[\begin{array}{rrrr}
-1 & 0 & 0 & 0 \\
0 & -2 & 0 & 0 \\
0 & 0 & -3 & 0 \\
0 & 0 & 0 & -4
\end{array}\right] \mathbf{x}=\left[\begin{array}{l}
1 \\
1 \\
1 \\
1
\end{array}\right] \mathbf{u}+\mathbf{w} \\
\mathbf{y}=\left[\begin{array}{llll}
1 & 1 & 1 & 1
\end{array}\right] \mathbf{x}+\mathbf{v}
\end{gathered}
$$

with intensities $\mathbf{W}=10^{3} \mathbf{I}_{4}$ and $\mathbf{V}=1$ and weighting matrices $\mathbf{R}_{1}=10^{4} \mathbf{I}_{4}$ and $\mathbf{R}_{2}=1$. The optimal compensator is given by $\mathbf{K}^{\circ}=\left[\begin{array}{lllll}22 \cdot 20 & 15 \cdot 10 & 12 \cdot 56 & 11 \cdot 21\end{array}\right]^{\mathrm{T}}$ and $\mathbf{F}^{\circ}=[71.0148 .8041 .0236 .97]$ and its transfer function is

$$
C^{\mathrm{o}}(s)=\frac{3243(s+3 \cdot 753)(s+2 \cdot 693)(s+1 \cdot 626)}{(s+264 \cdot 49)(s+3 \cdot 504)(s+2 \cdot 267)(s-1 \cdot 397)}
$$

which is unstable. The optimal cost is $7 \cdot 2156 \times 10^{6}$.

First we look for the stable suboptimal full-order compensator. With $\alpha=0$ and $\rho=0 \cdot 0014$ equation (11) has a non-negative definite solution, i.e. the corresponding state feedback $\mathbf{F}=\left[\begin{array}{llll}2 \cdot 23 & 2 \cdot 26 & 2 \cdot 25 & 2 \cdot 25\end{array}\right] \times 10^{3}$ leads to a stable compensator. The cost is $8 \cdot 2236 \times 10^{6}$. The final tuning procedure of (13) gives, for $\lambda=5 \cdot 5 \times 10^{-4}$, a controller with one pole at the origin and a cost of $7 \cdot 2215 \times 10^{6}$. The increase in cost is therefore negligible, less than $0 \cdot 1$ per cent.

The optimal second-order controller for this system is also unstable and leads to the cost $7 \cdot 2157 \times 10^{6}$, practically the same as the optimal cost. To find a stable reduced-order compensator, we choose $\rho_{1}=\rho_{2}=\rho_{3}=0.001$ and follow the procedure outlined at the end of Section 3. For $\lambda_{1}=\lambda_{2}=4.05 \times 10^{-8}$ we get

$$
C(s)=\frac{531(s+2 \cdot 02)}{s(s+307 \cdot 6)}
$$

and the cost is $7 \cdot 2375 \times 10^{6}$, again a negligible increase. If one wishes to have a strictly stable controller, then increasing $\lambda_{1}$ and $\lambda_{2}$ to $8 \times 10^{-8}$ yields a controller with a slowest pole at -0.45 with only 0.6 per cent increase in cost. When the costs are practically equal, it is reasonable to assume that a second-order stable controller is preferable to a fourth-order unstable one. Incidentally, this example demonstrates a situation which is not uncommon in optimization schemes. In the parameter space there might be directions along which the cost function is relatively flat. Large variations of the parameters in those directions can be used to achieve further performance specifications, such as stability of the controller in this case, with only a small increase in cost.

\section{DISCUSSION}

The problem of stable LQG controllers was considered. By modifying the state weighting matrix and/or the process noise intensity matrix, stable controllers were achieved for both the 
full-order and reduced-order cases. The modifications are implicit in the sense that they depend on the new value of $\mathbf{P}$ in the full-order case or the new values of $\mathbf{Q}, \mathbf{P}$ and $\hat{\mathbf{P}}$ in the reducedorder case. Consequently the generalized Riccati equations that have to be solved have a generally indefinite matrix coefficient of the quadratic term as in $H_{\infty}$-theory.

A final tuning procedure is used to minimize the deviation from the original cost function, which in turn normally means a reduction in the cost evaluated from the original cost function. The sufficient conditions in Theorem 1 and 4 give controllers with poles which are generally well into the LHP. The final tuning procedure pushes the unstable poles of the original optimal controller towards the LHP and stops when the last of these poles reaches the imaginary axis. Hence applying this procedure always results in a controller which is on the verge of instability. Such controllers often lead to conditionally stable systems. ${ }^{9}$ To avoid such undesirable designs, $\lambda>\lambda^{*}$ may be used, with the idea that a system with all its open-loop poles well into the LHP is less likely to be conditionally stable.

\section{ACKNOWLEDGEMENTS}

Y.H. was supported in part by the Technion V.P.R. Fund-E. \& J. Bishop Fund.

\section{REFERENCES}

1. Youla, D. C., J. J. Bongiorno and C. N. Lu, 'Single loop feedback-stabilization of linear multivariable dynamical plants', Automatica, 10, 159-173 (1974).

2. Bernstein, D. A., and D. C. Hyland, 'The optimal projection equations for reduced order state estimation', IEEE Trans. Automatic Control, AC-30, 583-585 (1985).

3. Hyland, D. C. and D. S. Bernstein, 'The optimal projection equations for fixed-order dynamic compensation', IEEE Trans. Automatic Control, AC-29, 1034-1037 (1984).

4. Haddad, W. M. and D. S. Bernstein, 'The optimal projection equations for reduced order state estimation: the singular measurement noise case', IEEE Trans. Automatic Control, AC-32, 1135-1139 (1987).

5. Halevi, Y., 'The optimal reduced-order estimator for systems with singular measurement noise', IEEE Trans. Automatic Control, AC-34, 777-781 (1989).

6. Halevi, Y. W., W. M. Haddad and D. S. Bernstein, 'A Riccati equation approach to the singular LQG problem', Proc. Am. Control Conf., Pittsburgh, PA, 1989.

7. Bernstein, D. S., and W. M. Haddad, 'LQG control with an $H_{\infty}$ performance bound; a Riccati equation approach', IEEE Trans. Automatic Control, AC-34, 293-305 (1989).

8. Feurer, T. M. and Y. Halevi, 'Stable suboptimal PQG controllers', Proc. I8th Ann. Pittsburgh Conf. of Modeling and Simulation, Pittsburgh, PA, 1987.

9. Ganesh, C., and J. B. Pearson, ' $H^{2}$-optimization with stable controllers', Automatica, 25, 629-634 (1989).

10. Halevi, Y., 'Design of stable suboptimal LQG controllers', Proc. Am. Control Conf., Minneapolis, MN, 1987.

11. Kwakernaak, H., and R. Sivan, Liner Optimal Control Systems, Wiley-Interscience, New York, 1972.

12. Doyle, J. C., and G. Stein, 'Robustness with observers', IEEE Trans. Automatic Control, AC-23, 607-611 (1979). 